\title{
Retained Digital Flexible Ureteroscope During Percutaneous Nephrolithotomy
}

\author{
Tony T. Chen, MD, Ian Metzler, MD, MEng, and Robert M. Sweet, MD
}

\begin{abstract}
Background: During percutaneous nephrolithotomy, retrograde flexible ureteroscopy can be utilized to facilitate repositioning of stones, enable direct vision percutaneous access, minimize radiation exposure, reduce operating times, and improve stone-free rates. Although advancements in technique and flexible ureteroscope technology for the past decades have rendered complications rare, herein we report a case of a retained ureteroscope during percutaneous nephrolithotomy that was effectively managed endoscopically.

Case Presentation: A 59-year-old Caucasian gentleman with a history of recurrent bilateral nephrolithiasis presents for a left-sided percutaneous nephrolithotomy for a large stone burden $>4 \mathrm{~cm}$. A ureteral access sheath was used and retrograde ureteroscopy was performed to first reposition several stones into the renal pelvis. During manipulation, we were unexpectedly unable to retract the ureteroscope from the access sheath. We describe procedural details leading up to the event and subsequent intraoperative management using an antegrade approach.

Conclusion: A retained flexible ureteroscope is a rare but serious intraoperative complication that may require invasive open surgical management. However, careful endoscopic management may be feasible in select cases, allowing for preservation of ureter and instrument.
\end{abstract}

Keywords: percutaneous nephrolithotomy, ureteroscopy, retained ureteroscope, complication

\section{Introduction and Background}

W ITH THE INCREASED PREVALENCE of stone disease, ureteroscope utilization has expanded in the surgical management of stone disease for the past several decades. A combined approach of ureteroscopy and percutaneous access in addressing larger volumes of stone disease has provided several advantages, including enabling the repositioning of stones, direct vision of percutaneous access, while also minimizing radiation exposure, reducing operating times, and improving stone-free rates. ${ }^{1}$

Although ureteroscope technological innovations and advancements in technique have rendered devastating intraoperative complications such as ureteral avulsion rare, the incidence of other injuries such as mucosal erosion, false passage, and perforation has been reported in a range up to $4 \%$ to $10 \%{ }^{2}$ A retained ureteroscope is even a rarer complication of ureteroscopy, with only a handful of cases reported in the literature. ${ }^{2}$ Herein, we describe a complication of a retained ureteroscope during retrograde endoscopy-assisted percutaneous nephrolithotomy (PCNL).

\section{Presentation of Case}

The patient is a 59-year-old Caucasian man with recurrent nephrolithiasis who presented for a planned left percutaneous nephrolithotomy at a tertiary academic medical center. Preoperative CT imaging demonstrated significant left-sided stone disease, $>4 \mathrm{~cm}$ in total burden, with multiple stones in the lower pole extending into the interpolar, upper pole calices without hydroureteronephrosis (Fig. 1). Under general anesthesia, the patient was positioned in split leg prone positioning and after retrograde access to the left kidney was obtained, a 12/14F $35 \mathrm{~cm}$ ureteral access sheath was placed

Department of Urology, University of Washington, Seattle, Washington, USA. 


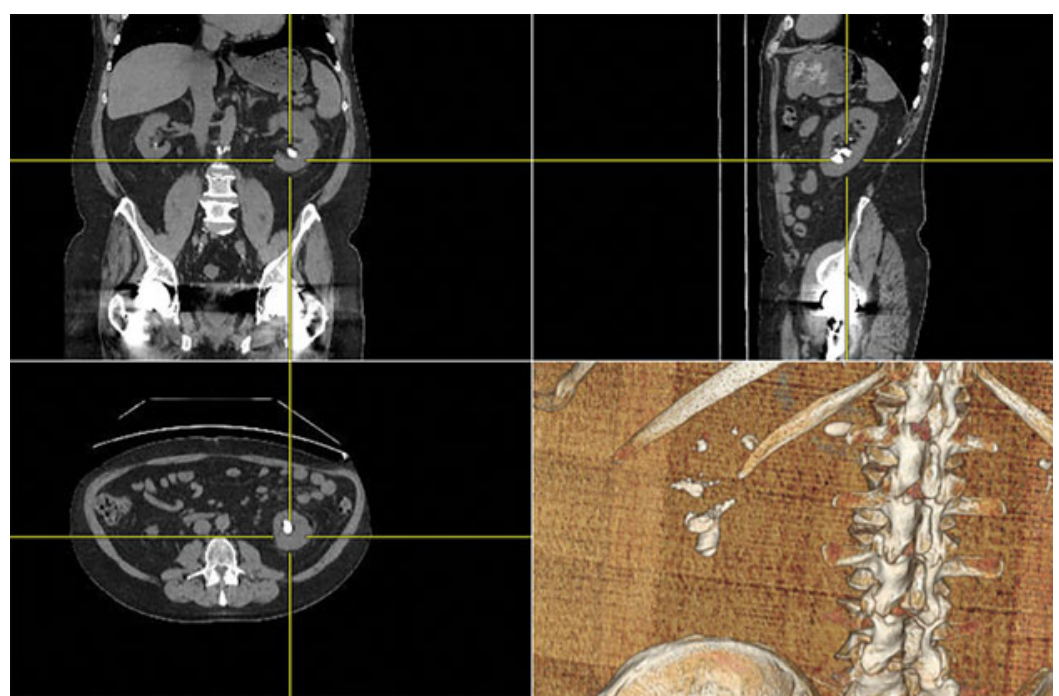

FIG. 1. Preoperative CT imaging demonstrating left stone burden in lower, interpolar, and upper pole calices.

(Fig. 2). A retrograde pyelogram was first performed and saved as a road map, demonstrating fine long infundibulum, a nondilated renal pelvis, and multiple filling defects within the lower, interpolar, and upper poles consistent with known stone burden (Fig. 3).

A digital flexible ureteroscope (Olympus Corporation, Center Valley, PA) was introduced into the kidney through the access sheath without any difficulty and no deficiencies in deflection were noted. Numerous stones 1 to $17 \mathrm{~mm}$ in size were encountered and repositioned into the renal pelvis with a Halo $1.5 \mathrm{~F}$ basket (Sacred Heart, Minnetonka, MN). During this step, it was noted that the ureteroscope could not be retracted from the ureteral access sheath. After contrast instillation for interrogation, the kidney appeared to move with gentle attempts to manipulate and remove the ureteroscope.

At this point, a pause was held and endourologic $v s$ open surgical approaches were discussed-given there was no contrast extravasation and low concern for ureteral perforation, we decided to proceed further with endoscopic inves-

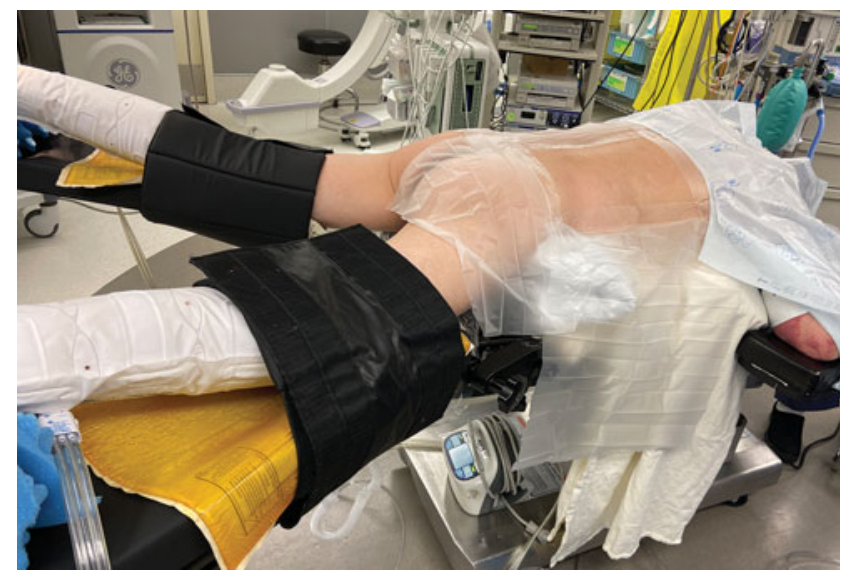

FIG. 2. Patient positioning during retrograde endoscopyassisted percutaneous nephrolithotomy. tigation in an antegrade approach. Antegrade percutaneous lower pole access with standard bull's eye technique was obtained and a 24F nephrostomy sheath was placed with balloon dilation. Antegrade flexible ureteroscopy with a second ureteroscope directly adjacent to the existing ureteroscope revealed significant steinstrasse. Specifically, dozens of 2 to $5 \mathrm{~mm}$ stones were tightly clustered and impacted immediately distal to the tip of the retained ureteroscope in the upper ureter. These stones remained proximal to the access sheath but prevented the retained ureteroscope's removal or manipulation (Fig. 4).

Most of the PCNL was then completed carefully with a dual action lithotrite (Olympus Corporation) and the stones

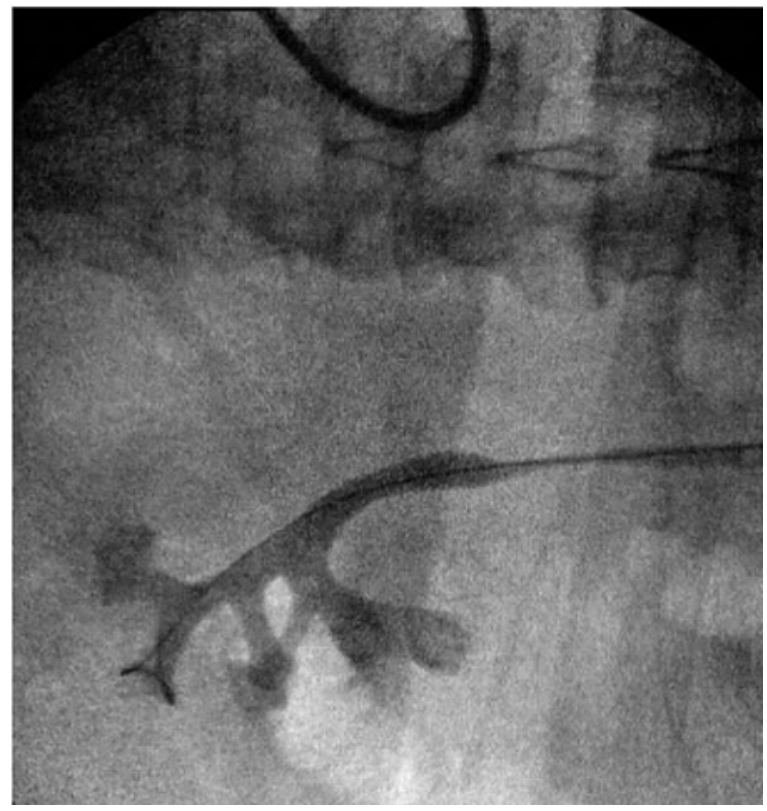

FIG. 3. Initial retrograde pyelogram demonstrating a nondilated left renal pelvis, long infundibulum, and multiple filling defects consistent with stone disease. 


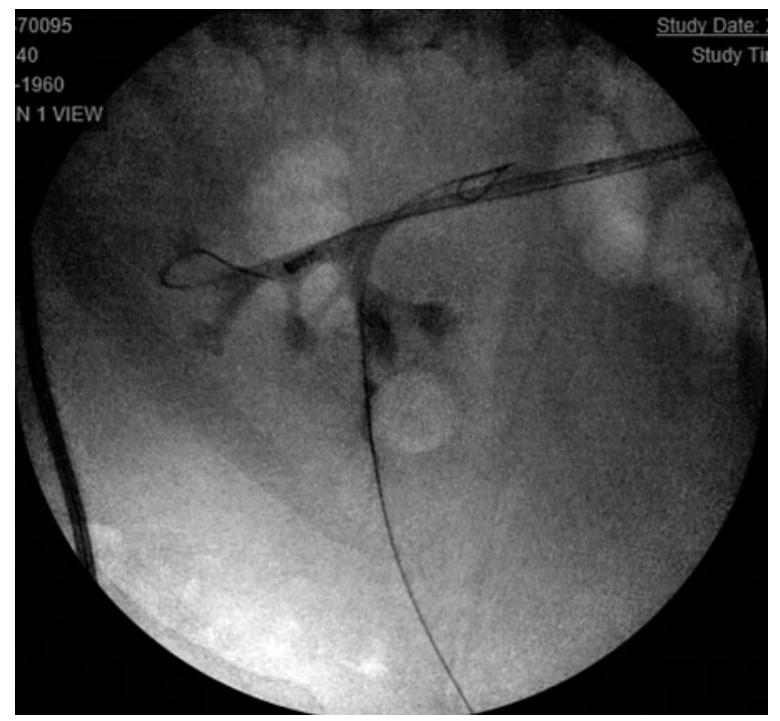

FIG. 4. Antegrade percutaneous access in left posterior lower pole calix to access and evaluate retained ureteroscope seen within a ureteral access sheath.

clustered around the retained ureteroscope were effectively basketed antegrade with a Halo $1.5 \mathrm{~F}$ basket using the second ureteroscope. The retained ureteroscope was then easily retracted out of the access sheath and there were no evident mechanical or functional defects, including deflection on thorough evaluation of the ureteroscope. The ureteroscope was reinserted into the access sheath and several remaining fragments near the ureteropelvic junction were basketed retrograde uneventfully. On pullback ureteroscopy, the ureter was completely intact with no evidence of mucosal erosion, submucosal tunneling, or ureteral perforation. The patient was stone free at the conclusion of the procedure and had an uneventful postoperative course. He was discharged postoperative day 1 with a stent and suffered no additional complications.

\section{Discussion and Literature Review}

Although retained ureteroscopes from endoscopic management of stone disease are exceedingly rare, two cases have been reported in the literature. In both instances, the outer shaft skin of the ureteroscope was damaged, forming folds that created an accordion-like defect with attempts at withdrawal and manipulation. In the first case, a ureteroscope was unable to be retracted after laser lithotripsy of a $10 \mathrm{~mm}$ proximal right ureteral stone. Nonsurgical management was initially attempted and the retained ureteroscope was left in situ for 48 hours, allowing for passive dilation. Despite so, the ureteroscope was unable to be retracted on subsequent attempt. The patient was transferred to a tertiary academic medical center, where orthopedic bolt cutters were used to transect the ureteroscope at the level of the hand piece. An access sheath $12 / 14$ was passed over the retained tip to eliminate the accordion defect and the ureteroscope was uneventfully removed. ${ }^{2}$
In the second case, a ureteroscope was unable to be retracted after laser lithotripsy of a $1.1 \mathrm{~cm}$ proximal left ureteral stone. Ultimately, bolt cutters were used to transect the ureteroscope and the patient underwent open surgery for removal of retained ureteroscope wires and fiber optics. As in the first case, the ureteroscope had a rippling defect in the outer skin, creating an accordion-like effect with manipulation. $^{2}$

Although the mechanism accounting for the retained ureteroscope in our case is different from those previously reported, overlooked ureteroscope deficiencies may lead to unanticipated complications, including a retained ureteroscope. Although the durability of ureteroscopes is affected by design, surgeon experience, and technique of use, previous studies suggest a significant deterioration in ureteroscope performance characteristics after repetitive ureteroscopy. Specifically, active deflection decreased by as much as $28 \%$ after 6 to 15 uses, and on average, a ureteroscope required repair after 10 to 34 uses. $^{3}$ Therefore, it remains important for urologists to inspect ureteroscopes before use for outer shaft skin integrity and deficiencies in deflection or video output.

Preliminary studies demonstrate single-use ureteroscopes as comparable with nondisposable ureteroscopes in manipulation and visibility, and may even reduce operating room time and complications - although the adaptation of such ureteroscopes in clinical practice are low, consideration of such single-use ureteroscopes can help prevent complications leading to a retained ureteroscope. ${ }^{4}$

In our case, it was not a ureteroscope defect that led to the inability to retract the ureteroscope but rather steinstrasse of small stones that migrated distal to the tip of the ureteroscope toward the ureteropelvic junction. This is an important consideration to be anticipated for all ureteroscopy cases, especially for cases involving a large volume of stone disease and a combined ureteroscopy and percutaneous access approach. In particular, it is important for urologists to remain vigilant when endoscopically maneuvering past stones in the collecting system to access proximal sites for visually assisted percutaneous puncture.

\section{Conclusion}

A retained ureteroscope remains an exceedingly rare complication of ureteroscopy during endoscopic management of stone disease. Although mechanisms leading up to the event may vary, simple preventive measures such as thorough inspection of ureteroscope before insertion, use of disposable ureteroscopes, and guarding the ureteropelvic junction from gathering large stone volume is encouraged to eliminate the risk of such a complication altogether. In the case of steinstrasse leading to ureteroscope retention, percutaneous access and antegrade ureteroscopy to remove impacted stones surrounding a ureteroscope can address such a complication without additional invasive procedures or loss of costly instruments.

\section{Disclosure Statement}

No competing financial interests exist. 


\section{Funding Information}

No funding was received for this article.

\section{References}

1. Alsyouf M, Arenas JL, Smith JC, et al. Direct endoscopic visualization combined with ultrasound guided access during percutaneous nephrolithotomy: A feasibility study and comparison to a conventional cohort. J Urol 2016;196: 227-233.

2. Huynh M, Telfer S, Pautler S, Denstedt J, Razvi H. Retained digital flexible ureteroscopes. J Endourol Case Rep 2017;3: 24-27.

3. User HM, Hua V, Blunt LW, Wambi C, Gonzalez CM, Nadler RB. Performance and durability of leading flexible ureteroscopes. J Endourol 2004;18:735-738.

4. Usawachintachit $M$, Isaacson DS, Taguchi $K$, et al. A prospective case-control study comparing LithoVue, a single-use, flexible disposable ureteroscope, with flexible, reusable fiber-optic ureteroscopes. J Endourol 2017;31: 468-475.
Address correspondence to: Robert M. Sweet, MD Department of Urology University of Washington 1959 NE Pacific Street Box 356410

Seattle, WA 98195

USA

E-mail: rsweet@uw.edu

\section{Abbreviations Used \\ $\mathrm{CT}=$ computed tomography \\ PCNL $=$ percutaneous nephrolithotomy}

Cite this article as: Chen TT, Metzler I, Sweet RM (2020) Retained digital flexible ureteroscope during percutaneous nephrolithotomy, Journal of Endourology Case Reports 6:3, 184-187, DOI: 10.1089/ cren.2020.0010. 